\title{
NOTES
}

\section{Anisotropic Structure Formed in Atactic Polystyrene Gels}

\author{
Xu-Ming XIE, Akihiko TAnIOKA, and Keizo MiYASAKA \\ Department of Organic and Polymeric Materials, \\ Tokyo Institute of Technology, Ookayama, \\ Meguro-ku, Tokyo 152, Japan
}

(Received August 19, 1987)

\begin{abstract}
KEY WORDS Anisotropic Structure / Atactic Polystyrene / Carbon Disulfide / Gel / Polarizing Microscope / IR Spectrum / Sheaf-Like Spherulite /
\end{abstract}

Some crystalline polymers form thermoreversible gels from their solutions at low temperature. These gels are three dimensional networks of chains cross-linked by physical bonds $^{1,2}$ which relate more or less with the crystallization. Wellinghoff $e t a l .^{3}$ and Tan $e t$ $a l^{4}{ }^{4}$ recently reported that non-crystallizable atactic polystyrene (at-Pst) makes gels with many kinds of solvents. They ${ }^{4}$ and Boyer et $a l^{5}$ investigated in detail the at-Pst gels as functions of molecular weight of polymer, concentration of polymer in the solvents and species of solvent, and proposed that the gelation is a second order transition occurring at $T_{\text {II }}$ which is a characteristic temperature higher than the glass transition point. According to them, the gelation arises from segment-segment interaction. On the other hand, an enhanced low angle light scattering for at-Pst solution was observed by Guenet et al ${ }^{6,7}$ Further they studied the endotherm of the gels in $\mathrm{DSC}^{8}$ and suggested that at-Pst/ $\mathrm{CS}_{2}$ solution forms a polymer-solvent complex or the stoichiometric compound. As seen in this review, there is still a serious ambiguity in the gelation mechanism of at-Pst solutions. In order to elucidate the mode of gelation and the structure of the gels, we investigated the morphology and properties of at-Pst $/ \mathrm{CS}_{2}$ gels. ${ }^{9}$ In the course of study, it was found that not a small number of patches with an anisotropic structure are formed in the uniform gel matrix. This anisotropic patches disappear when the gel melts on heating, but survive in the dried gel. However, this anisotropic patches never appear in the solvent-cast films, indicating that the gelation is necessary for their origination.

\section{EXPERIMENTAL}

Three commercially available samples of atPst were used in this study. The molecular weights of the samples are shown in Table I.

The at-Pst $/ \mathrm{CS}_{2}$ solutions the polymer concentrations of which were from $20 \%$ to $60 \%$ were prepared in sealed glass tubes with an inner diameter of $12 \mathrm{~mm}$. After the polymer was dissolved in $\mathrm{CS}_{2}$, the solution was kept standing at $40^{\circ} \mathrm{C}$ for one day. Then, it was quenched in a freezer kept at $-20^{\circ} \mathrm{C}$ for $48 \mathrm{~h}$ to make a gel. Dried gel films were made in the following way: first the solutions in shallow glass dishes were cooled to form gels. The gels were kept standing at the gelation temperature for more than 2 days, during which the solvent evaporated freely. Gel films thus dried were further dried under vacuum of $c a \cdot 10^{-2} \mathrm{mmHg}$ for $5 \mathrm{~h}$ at room temperature. The IR spectra confirmed that dried gel films thus obtained 
had not any noticeable amounts of solvent. At-Pst films were prepared by casting from the $\mathrm{CS}_{2}$ solution at room temperature, and from the tetrahydrofuran and toluene solutions at $-20^{\circ} \mathrm{C}$. No gelation took place under these conditions. The morphology of gels was observed using an Olympus model BHSP polarizing microscope. Infrared spectra of at-Pst dried gel films and solution-cast films were recorded on a Jasco model FT-IR3 Fourier transform spectrometer. Five hundred co-added interferograms at a resolution of $2 \mathrm{~cm}^{-1}$ were used to obtain each spectrum.

Further, a Shimadzu model FTIR-4100 Fourier transform spectrometer was used to obtain each microscopic spectrum of localized particular structure and homogeneous matrix of gel. The IR spectra were recorded using a resolution of $4 \mathrm{~cm}^{-1}$ in this case.

Table I. Molecular weights of at-PS samples

\begin{tabular}{cccc}
\hline & $\bar{M}_{n} \times 10^{4}$ & $\bar{M}_{w} \times 10^{4}$ & $\bar{M}_{w} / \bar{M}_{n}$ \\
\hline PS-A & 12.34 & 20.66 & 1.67 \\
PS-B & 15.21 & 26.37 & 1.73 \\
PS-C & 16.85 & 30.11 & 1.79 \\
\hline
\end{tabular}

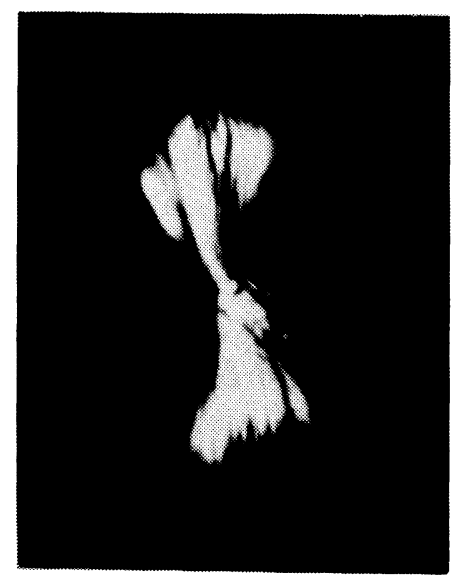

\section{RESULTS AND DISCUSSION}

At-Pst/CS $\mathrm{CS}_{2}$ solutions made gels on cooling, and the gel melted on heating, as already reported. These transitions are thermoreversible. When the gel was taken out of the freezer and immediately put on the stage of microscope, one could see a large number of bright patches existing in the gel under crossed nicols. These patches with particular structure appeared in the gels from the solutions of all the Pst samples used and all the polymer con-

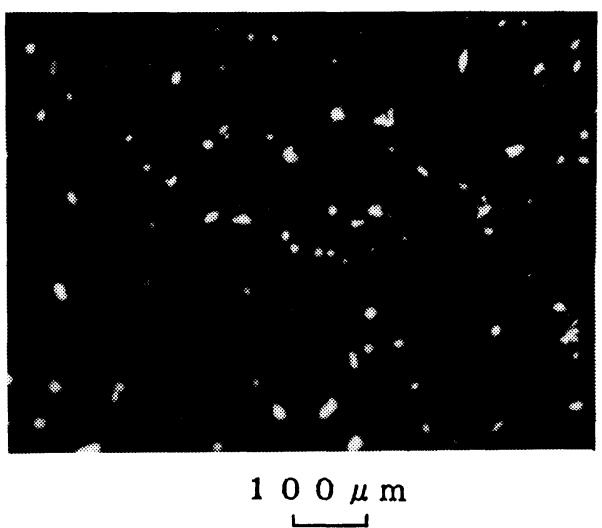

Figure 1. A microscopic photograph of at-Pst/CS dried gel under crossed nicols.

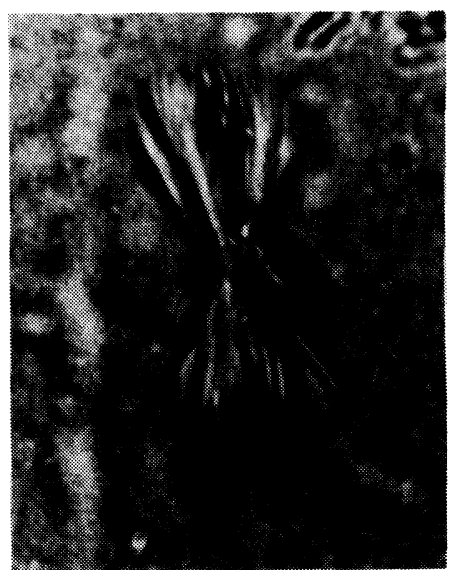

$10 \mu \mathrm{m}$

Figure 2. A microscopic photograph of a patch of sheaf-like shape formed in at-Pst/CS dried gels. (a) under crossed nicols; (b) without crossed nicols. 
centrations from $20 \mathrm{wt} \%$ to $60 \mathrm{wt} \%$ in the solutions. The anisotropic structure could survive in dried gels, as shown in Figure 1. One can see a number of bright patches in the photograph of a dried gel under crossed nicols. Most of the patches are as large as $10 \mu \mathrm{m}$ long. On the other hand, this type of patches was never observed in the films cast from the solutions at high temperatures where no gelation took place. This means that the gelation is necessary for the origination of the patch structure.

Figure 2 shows that the each patch has sheaf-like shape which is seen widely in polymer spherulites. When dried gels were warmed on the stage of microscopy at a heating

(a)

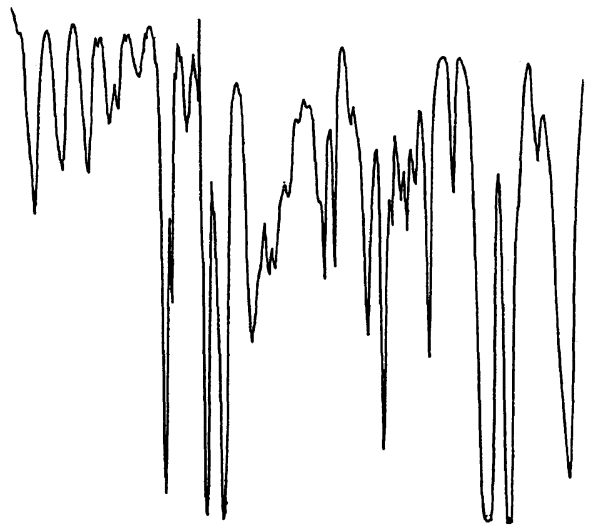

(b)

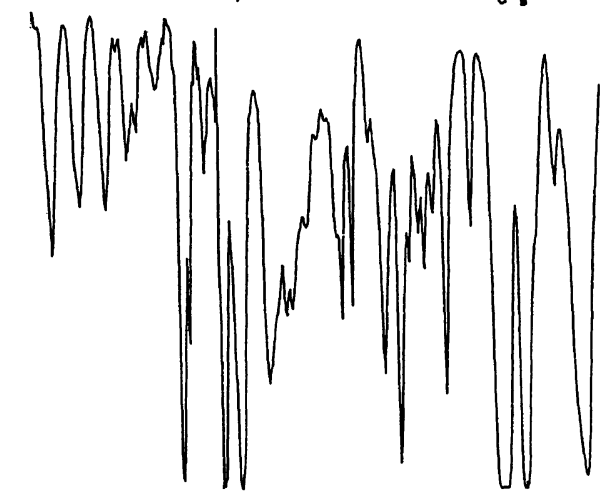

2000

$500 \mathrm{~cm}^{-1}$

Figure 3. (a) Infrared spectrum of an at-Pst/ $/ \mathrm{CS}_{2}$ dried gel film.

(b) Infrared spectrum of an at-Pst $/ \mathrm{CS}_{2}$ solution cast film at room temperature. rate of $10^{\circ} \mathrm{C} \mathrm{min}^{-1}$, the anisotropic structure vanished at $60^{\circ} \mathrm{C}$. When the dried gel was heated up and then cooled, the patches appeared again at $55^{\circ} \mathrm{C}$, unless the heating was over about $100^{\circ} \mathrm{C}$, the glass transition point of at-Pst. No further growth of the patches in dried gels took place during heating, which indicates that the origination and growth have a high correlation with the gelation. Figure 3 illustrates the IR spectra of a dried gel and solution cast film. These spectra only show

(a)

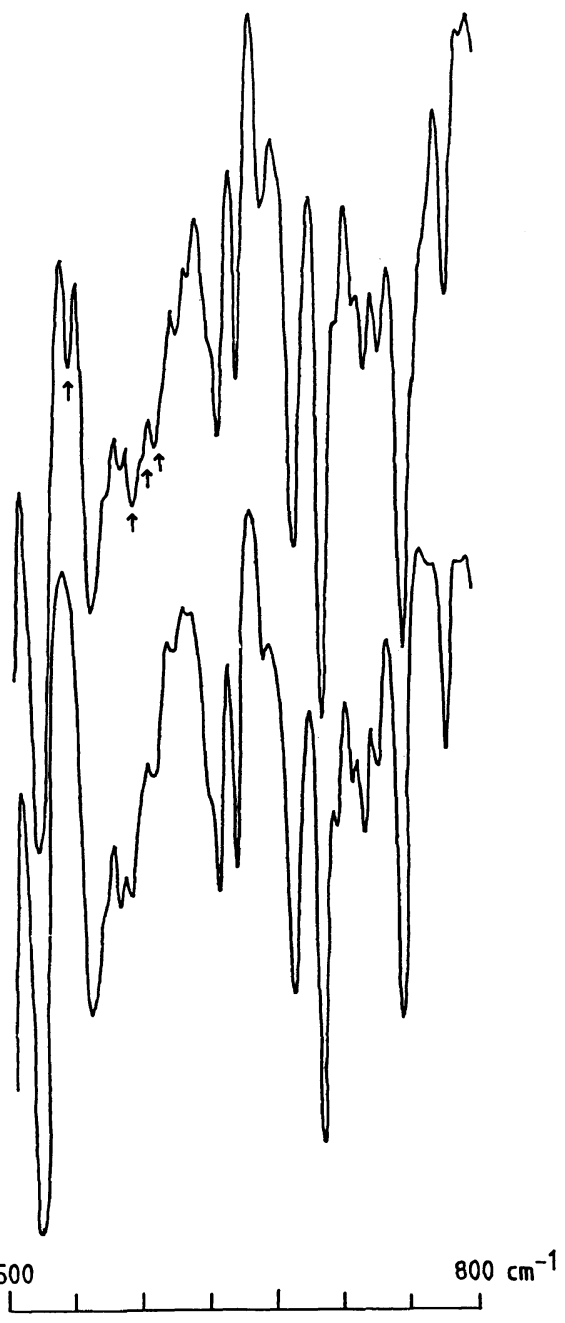

Figure 4. (a) Microscopic FT-IR spectra focused on an anisotropic patch in an at-Pst/ $\mathrm{CS}_{2}$ dried gel film.

(b) That focused on an isotropic matrix in the same dried gel film. 
that they are of atactic Pst, but do not show any substantial difference between them. In other wards, Figure 3 fails to distinguish the structure of the patches from that of the usual amorphous phase of the polymer. This may be due to that the fraction of the material making the patches is not large enough to be distinguished.

Figure 4(a) shows a microscopic IR spectrum obtained by focusing a patch in a dried gel, while Figure 4(b) shows that focused on the isotropic matrix region in the same sample. The comparison of these spectra indicates that the absorption intensities of bands at 1408, 1313,1297 , and $1185 \mathrm{~cm}^{-1}$ are larger in Figure 4(a) than in Figure 4(b). At this moment, we cannot assign these bands in the microscopic IR spectra. However, it should be remarked that these changes in IR spectra except $1408 \mathrm{~cm}^{-1}$ band have also been observed in crystallization of isotactic-PS. ${ }^{10}$ This microscopic IR spectrum of the patch in a dried gel together with the sheaf-like shape strongly suggest that the patches have a anisotropic structure like spherulite of crystalline polymers. It may be possible to suppose that there is a tacticity distribution along each chain, and that some segments, in which iso or syndio population is rich, gather together to make small crystalline or liquid crystalline fragments. However, it is difficult to suppose that such small fragmental elements can form a spherulitic structure as large as $10 \mu \mathrm{m}$ in the length. Further, the $1408 \mathrm{~cm}^{-1}$ band in the microscopic IR must be assigned before we reach the final conclusion.

The structure of the patches is now being investigated, and the result will be shown in the following paper.

\section{REFERENCES}

1. P. G. de Gennes, "Scaling Concept in Polymer Physics," Cornell University Press, Ithaca, New York and London, 1979.

2. P. J. Flory, Faraday Discuss. Chem. Soc., 57, 7 (1974).

3. S. T. Wellinghoff, J. Show, and E. Bear, Macromolecules, 12, 932 (1978).

4. H. Tan, A. Hiltner, E. Moet, and E. Bear, Macromolecules, 16, 28 (1983).

5. R. F. Boyer E. Bear, and A. Hiltner, Macromolecules, 18, 427 (1985).

6. J. M. Guenet, N. F. F. Willmott, and P. A. Ellsmore, Polym. Commun., 24, 230 (1983).

7. J. Y. S. Gan, J. Francois, and J. M. Guenet, Macromolecules, 19, 173 (1986).

8. J. Francois, J. Y. S. Gan, and J. M. Guenet, Macromolecules, 19, 2755 (1986).

9. X.-M. Xie, A. Tanioka, and K. Miyasaka, The annual meeting of the Society of Fiber Science and Technology, Japan, preprint, 169 (1987).

10. H. Tadokoro, N. Nishiyama, S. Nozakura, and S. Murahashi, J. Polym. Sci., 36, 553 (1959). 